Subscriber access provided by Uppsala universitetsbibliotek

\title{
Article
}

\section{Formation of deposition patterns induced by the evaporation of the restricted liquid}

Fushuai Wang, Mengmeng Wu, Xingkun Man, and Quanzi Yuan

Langmuir, Just Accepted Manuscript • DOI: 10.1021/acs.langmuir.0c01116 • Publication Date (Web): 01 Jul 2020

Downloaded from pubs.acs.org on July 2, 2020

\section{Just Accepted}

"Just Accepted" manuscripts have been peer-reviewed and accepted for publication. They are posted online prior to technical editing, formatting for publication and author proofing. The American Chemical Society provides "Just Accepted" as a service to the research community to expedite the dissemination of scientific material as soon as possible after acceptance. "Just Accepted" manuscripts appear in full in PDF format accompanied by an HTML abstract. "Just Accepted" manuscripts have been fully peer reviewed, but should not be considered the official version of record. They are citable by the Digital Object Identifier (DOI®). "Just Accepted" is an optional service offered to authors. Therefore, the "Just Accepted" Web site may not include all articles that will be published in the journal. After a manuscript is technically edited and formatted, it will be removed from the "Just Accepted" Web site and published as an ASAP article. Note that technical editing may introduce minor changes to the manuscript text and/or graphics which could affect content, and all legal disclaimers and ethical guidelines that apply to the journal pertain. ACS cannot be held responsible for errors or consequences arising from the use of information contained in these "Just Accepted" manuscripts. 


\title{
Formation of deposition patterns induced by the evaporation of the restricted liquid
}

\author{
Fushuai Wang ${ }^{1,2}$, Mengmeng Wu ${ }^{3,4}$, Xingkun Man ${ }^{3,4}$, Quanzi Yuan ${ }^{1,2 *}$ \\ ${ }^{1}$ State Key Laboratory of Nonlinear Mechanics, Institute of Mechanics, Chinese Academy of \\ Sciences, Beijing 100190, People’s Republic of China \\ ${ }^{2}$ School of Engineering Science, University of Chinese Academy of Sciences, Beijing 100049, \\ People's Republic of China \\ ${ }^{3}$ Center of Soft Matter Physics and its Applications, Beihang University, Beijing 100191, China \\ ${ }^{4}$ School of Physics, Beihang University, Beijing 100191, China
}

\begin{abstract}
AUTHOR INFORMATION
Corresponding Author

*E-mail: yuanquanzi@lnm.imech.ac.cn
\end{abstract}

Author Contributions

The manuscript was written through contributions of all authors. All authors have given approval to the final version of the manuscript. 


\begin{abstract}
Evaporation-induced self-assembly of colloids or suspensions has received increasing attention. Given its critical applications in many fields of science and industry, we report deposition patterns constructed by the evaporation of the restricted aqueous suspension with polystyrene particles at different substrate temperatures and geometric container dimensions. With the temperature increases, the deposition patterns transition from honeycomb to multi-ring to island, which is attributed to the competition between the particle deposition rate $U_{P}$ and the contact line velocity $U_{C L}$. And the dimension of the geometric container has an effect on the characteristics of patterns. In this paper, the formation of ordered multi-ring pattern is mainly focused on as a result of $U_{P}$ can keep up with $U_{C L}$, so that the entire contact line can be pinned, that is, the periodic stick-slip motion of the contact line and the particle sedimentation. Moreover, based on the Onsager principle, we develop a theoretical model to reveal the physical mechanisms behind the multi-ring phenomena. The position and spacing of rings are measured, which shows that the theoretical prediction agrees well with experiments. We also find that the ring spacing decays exponentially from center to edge experimentally and theoretically. This may not only help to understand the formation of the deposition patterns, but also assist future design and control in practical applications.
\end{abstract}

\title{
KEYWORDS
}

Deposition patterns, Evaporation, Stick-slip motion, Particle sedimentation, Onsager principle 


\section{INTRODUCTION}

How to construct various deposition patterns has received increasing attention in scientific research [1,2], due to its important applications in the fields of phononics [3], optical devices [4], components separation [5], bioscience [6, 7], crystallization [8], etc. Hence, kinds of methods have been developed, such as polymer-demixed [9], surface wrinkling [10], excimer laser processing [11]. Besides, as an important technology, evaporation-induced self-assembly has been studied extensively and has played a great role in many practical applications, such as spray cooling [12], printing [13] and self-assembly of particles [14]. More importantly, evaporation-induced selfassembly is easy to operate and low-cost, which is used to construct deposition patterns. For example, Deegan et al. studied the pattern of coffee-ring (i.e. a ring-like deposition left on the substrate after a coffee droplet evaporates) using a physical model during the evaporation of colloidal suspension droplet $[15,16]$. They attributed the formation of coffee-ring to capillary flow, a supplemental flow from center to edge induced by the intense evaporation loss at the triple phase contact line (TPCL) of droplet. Later, Hu et al. found that the condition for forming the coffeering pattern is that Marangoni flow at the liquid-vapor interface needs to be suppressed [17]. The formation of ring-like deposition is due to the pinning of TPCL and the intense evaporation loss at the vicinity of TPCL [18]. Therefore, the formation of multi-ring pattern needs to meet the condition, periodic motion of pinning and depinning of the TPCL, i.e. the stick-slip motion. However, the depinning of TPCL depends on miscellaneous factors, such as the size and the concentration of solute $[19,20]$, the property of substrate $[21,22]$. Weon et al. used $20 \mu \mathrm{m}$ diameter polystyrene particles dispersed in a water drop to construct a central deposition pattern 
[23]. The dendritic patterns were obtained by evaporating a droplet containing a mixture of $\mathrm{NaCl}$ solute and colloids [24]. And Yunker et al. used elliptical particles in a water drop to construct uniform deposition [2]. In these cases, the stick-slip motion of TPCL does not occur. The multiring pattern was obtained by evaporation of a sessile droplet of DNA solution [25]. In addition, by fixing the lower plate and controlling the sliding speed of the upper plate precisely, the polymer solution in two parallel plates could evaporate and form parallel stripes [26]. Restricting droplet to geometric container which controls the motion of the contact line helps to obtain ordered deposition patterns $[27,28]$. Liquid evaporation in geometric container, which provides a new path for construction of deposition patterns, has received increasing attention. However, as far as we are concerned, little work has been done to study the dimension effect of the geometric container and the temperature effect of the substrate on the formation of the deposition patterns. In addition, due to the combined effects of internal flow field, contact line motion and solute transport, theoretical modeling of restricted liquid evaporation remains a challenge.

In this paper, deposition patterns formed by the evaporation of restricted liquid have been studied. Experiments were carried out under different temperatures $(T=293 \mathrm{~K}-383 \mathrm{~K})$, inner diameters $(D=2.5,4.5$ and $6.5 \mathrm{~mm})$ and heights $(L=2,4,6$ and $8 \mathrm{~mm})$, generating five types of patterns which are honeycomb, multi-ring, island and two kinds of transition patterns, respectively. A dimensionless parameter which can represent the competition between the particle deposition rate and the contact line velocity was used to analyze the formation of deposition patterns. The multiring pattern as an ordered structure is mainly focused on and based on the Onsager principle, the mechanism of multi-ring formation was explained. The position and spacing of rings were also 
analyzed experimentally and theoretically. Our findings not only give an uncomplex and low-cost method but also provide guidance for construction of deposition patterns.

\section{EXPERIMENT}

In our experiments, the monodispersed polystyrene (PS) latex microsphere aqueous suspension (0.5 $\mu \mathrm{m}, 2.5 \mathrm{wt} \%$, Alfa Aesar Chemical Co., Ltd, China) was diluted in deionized water to provide aqueous suspension. The aqueous PS suspension was selected in our experiments since it tends to deposit to form stable structures which is easy to observe [29 - 31]. The glass slides $\left(25 \times 75 \times 1.2 \mathrm{~mm}^{3}\right)$ and the hollow cylindrical tubes of various dimensions $(D=2.5,4.5,6.5$ $\mathrm{mm}, L=2,4,6,8 \mathrm{~mm}$, thickness: $1 \mathrm{~mm}$ ) are made of the same material, indicating the same wettability. Besides, they were cleaned ultrasonically with alcohol for twenty minutes and then left to rest for 3 minutes to ensure that the residual alcohol on the surface volatilizes completely. Figure 1 schematically shows the experimental setup. Cylindrical glass tube was vertically bonded to the glass slide by UV glue. To ensure that the distance from center of the concave liquid surface to substrate surface is a constant value under all experimental conditions as shown in Figure 1b, the volume of droplet was precisely controlled by pipette. The droplet volume was $6.0 \mu \mathrm{L}$ for the diameter of $2.5 \mathrm{~mm}, 19.5 \mu \mathrm{L}$ for the diameter of $4.5 \mathrm{~mm}$, and $40.6 \mu \mathrm{L}$ for the diameter of $6.5 \mathrm{~mm}$. The temperature was set to span a large range from $293 \mathrm{~K}$ to $383 \mathrm{~K}$ controlled by thermostatic heating plate. The evaporation process of restricted liquid was captured by a highspeed camera and the deposition patterns were photographed with a microscope. 


\section{RESULTS AND DISCUSSION \\ The transition of deposition patterns}

Figure 2a summarizes the deposition patterns formed by the evaporation of the restricted aqueous PS suspension at different substrate temperatures $(T=293 \mathrm{~K}-383 \mathrm{~K})$ and geometric container dimensions $(D=2.5,4.5,6.5 \mathrm{~mm}$ and $L=2,4,6,8 \mathrm{~mm})$. Obviously, there are five types of deposition patterns, honeycomb, multi-ring, island and two transition patterns. Note that temperature $T$ plays a decisive role in the formation of deposition patterns, which determines the evaporating rate in our experiments. The evaporating rate directly affects the particle deposition and the motion of contact line. The competition between the receding contact line and the particle deposition at the contact line is introduced to explain the formation of deposition patterns [32, 33]. Here, a dimensionless parameter, $\xi=U_{P} / U_{C L}$, where $U_{P}$ and $U_{C L}$ represent the particle deposition rate and the contact line velocity, respectively, is used to analyze the formation of patterns [32]. Figure 3, which is a schematic illustration based on the experimental analysis, shows how $\xi$ varies with $T$, using the case of $D=2.5 \mathrm{~mm}$ as an example. Obviously, it can be seen that the competition between $U_{P}$ and $U_{C L}$ is not monotonous as $T$ increases, which is the result of the joint action of the size and the concentration of solute, interaction between solutes and substrate, the porosity and number of layers of the deposition [32].

From $293 \mathrm{~K}$ to $308 \mathrm{~K}$, the deposition pattern is honeycombed. The low deposition rate leads to the decrease of solute density near the contact line. Hence, the resistance acting on the contact line is not large enough to pin the entire contact line [34]. The unpinned contact line continues to 
receding, eventually causing the contact line to bend. Eventually, the particles deposit along the contact line, forming honeycomb pattern after repetition many times.

From $323 \mathrm{~K}$ to $338 \mathrm{~K}$, the deposition pattern presents multi-ring which is mainly focused on in this paper. Within this temperature range, the particle deposition rate $U_{P}$ can keep up with the contact line velocity $U_{C L}$, so that the entire contact line can be pinned. Then, the particles deposit near the entire contact line, forming a single ring pattern. Finally, the multi-ring pattern is formed after periodic stick-slip motion of the contact line and particle sedimentation. The stick-slip motion of the contact line on the substrate and tube wall was observed in experiments, as shown in Figure 4. However, the contact line on the substrate is the focus of this paper. At time $t_{1}$, the contact line on the tube wall is pinned, and due to the loss of the liquid volume during evaporation, a flow which is the combination of the Marangoni convection along the liquid-vapor interface and the capillary convection along the tube wall generates inside the liquid, as shown in Figure 1b. As evaporation proceeds, a thin film forms on the substrate and then breaks. A new contact line on the substrate appears and is pinned, as shown at time $t_{2}$. At this moment, another flow which is the combination of the radical supplementary flow (capillary convection) along the substrate from edge to center and the Marangoni convection along the liquid-vapor interface appears inside the liquid due to the loss of the liquid volume is intense at the contact line [16]. And this flow carries PS particles to the contact line on the substrate and PS particles deposit at the vicinity of the pinned contact line. The pinning of contact line on the substrate and the loss of liquid volume flatten the restricted liquid and create a force that causes contact line on the substrate to recede and be pinned 
to the next position, as shown at time $t_{3}$. So does the motion of the contact line on the tube wall. The periodic stick-slip motion of the contact line on the substrate and particles deposit at the pinned contact line generate the multi-ring pattern. Furthermore, it is found that the contact line on the substrate and tube wall moves alternately, as shown in Figure 4b. We think this decoupling behavior is caused by the asymmetry of the temperature distribution and the geometry. The contact line on the substrate moves faster than it on the tube wall during the entire event, which results in the motion on the tube wall is in equilibrium with respect to the motion on the substrate. Fortunately, this finding provides strong evidence for the establishment of our theoretical model. Another thing to note that during each receding of the contact line on the substrate, the contact angle gradually increases to a fixed value, thus entering the next stick state. And the fixed value corresponding to each new stick state is greater than the last one, which is due to the existence of wall.

The spacing between neighboring rings is also measured, showing the form of exponential decay from center to edge. The multi-ring patterns under four different conditions are analyzed: $T=323$ $\mathrm{K}, D=4.5 \mathrm{~mm}, L=2 \mathrm{~mm} ; T=338 \mathrm{~K}, D=4.5 \mathrm{~mm}, L=4 \mathrm{~mm} ; T=308 \mathrm{~K}, D=6.5 \mathrm{~mm}, L=2 \mathrm{~mm}$ and $T=323 \mathrm{~K}, D=6.5 \mathrm{~mm}, L=4 \mathrm{~mm}$. Figure 5 a shows the variation of positions of the rings from center to edge. If we use the centesimal system to indicate the regions of rings, the first ring is about $50 \%$, the second ring is about $70 \%$, the third ring is about $80 \%$, the fourth ring is about $90 \%$ and the fifth ring is about $98 \%$. Besides, the spacing between neighboring rings gradually decreases exponentially from center to edge, as shown in Figure $5 \mathrm{~b}$. 
When $T=383 \mathrm{~K}$, the temperature exceeds the boiling point of the solvent makes the solvent evaporate quickly and triggers multiple eddies inside the liquid [35]. In this case, the contact line velocity $U_{C L}$ is much greater than the particle deposition rate $U_{P}$, resulting in partial contact line being pinned. During the receding process of the contact line, when it encounters the particles, deposition may occur near the contact line. Eventually, island pattern forms.

The remaining two deposition patterns, we define them as transition states. Obviously, these two transition states have opposite patterns from center to edge. In these cases, both entire and partial pinning of the contact line occur, resulting in the combination of radial spokes and ring.

Similar deposition pattern transitions are observed at $D=4.5 \mathrm{~mm}$ and $D=6.5 \mathrm{~mm}$, which can be seen from Figure 2a. Differently, there is no honeycomb pattern in both cases. And the ringlike pattern becomes smooth and ordered as $L$ decreases. $L$ affects the vapor pressure inside the container, which is an important factor in the generation of instability according to de Gennes's work [36]. It can be concluded that the instability near the contact line decreases as $L$ decreases. Besides, the multi-ring pattern is easier to form at $D=4.5 \mathrm{~mm}$ under $L$ set in the paper. We think that $D / L$, which affects the vapor pressure, may have an optimal range to form a stable pattern, such as multi-ring.

\section{Theoretical framework for multi-ring pattern}

As is known to all, stable deposition patterns can play an important role in many fields. In order to analyze the mechanisms of the formation of multi-ring pattern, a theoretical model is proposed to explain the evaporation process of the restricted liquid, including the motion of the contact line 
on the substrate, the evolution of the contact angle of the restricted liquid, etc. The schematic diagram of the theoretical model is shown in Figure 6.

The contact angle is small by using the lubrication approximation. Hence, the liquid-vapor interface profile of the restricted liquid is given by $h(r, t)=H(t)\left[r^{2} / R^{2}(t)-1\right]$, where $H(t)$ is the height of the extension of the parabola at the center of the substrate, and $R(t)$ is the radius of the contact line. Then, the contact angle $\theta$ is given by

$$
\theta=\frac{4 R V}{\pi\left(R^{2}-R_{0}^{2}\right)^{2}}
$$

where $V$ is the volume of the restricted liquid. It's worth noting that as $R$ approaches $R_{0}, \theta$ approaches infinity, that is, there is singularity. In our actual calculation, the calculation will be stopped when the volume $V$ is very small, to avoid this situation.

The analytic solution of $\dot{V}(t)$ can be obtained by solving the diffusion equation. Related studies $[37,38]$ have shown that $\dot{V}(t)$ is proportional to the radius of the contact line in the sessile droplet. Furthermore, due to the existence of geometric constraints surface, we assume

$$
\dot{V}(t)=\dot{V}_{0} \frac{\left[R_{0}^{2}-R(t)^{2}\right]^{\frac{1}{2}}}{R_{0}},
$$

where $\dot{V}_{0}(<0)$ is the initial rate of volume change, determined by the initial solid-liquid contact area and the environment. The evaporation flux, i.e. the rate of the evaporating volume, is given by

$$
J(t)=-\frac{\dot{V}_{0}}{\pi R_{0}\left(R_{0}^{2}-R^{2}\right)^{\frac{1}{2}}}
$$


Meanwhile, Eq. (3) indicates that the evaporating rate increases with the evaporation of restricted liquid.

Next, the evolution equation of $R(t)$ is derived based on the Onsager principle [39]. This principle is equivalent to the minimum energy dissipation principle in Stokesian hydrodynamics, which means that the evolution of the system is determined by the minimum of Rayleighian defined by $\mathfrak{R}=\Phi+\dot{F}$, where $\dot{F}$ is the time derivative of the free energy of the system, and $\Phi$ is the energy dissipation function.

The free energy $F$ is a sum of the interfacial energy, defined by

$$
\begin{aligned}
F= & \gamma_{L V} \int_{R}^{R_{0}} d r 2 \pi r \sqrt{1+h^{\prime 2}}+\gamma_{L S} \pi\left(R_{0}^{2}-R^{2}\right)-\gamma_{S V} \pi\left(R_{0}^{2}-R^{2}\right), \\
& +\gamma_{L S} 2 \pi R_{0} h\left(R_{0}\right)-\gamma_{S V} 2 \pi R_{0} h\left(R_{0}\right)
\end{aligned}
$$

where $\gamma_{L V}, \gamma_{L S}$ and $\gamma_{S V}$ are the liquid-vapor, liquid-sloid and solid-vapor interface tension, respectively. The first term represents the liquid-vapor interfacial energy. The second and third terms represent the interfacial energy on the substrate of liquid-sloid and solid-vapor, respectively. The fourth and fifth terms represent the interfacial energy on the tube wall of liquid-sloid and solidvapor, respectively.

As mentioned in the above experiment, the stick-slip motion of the contact line on the substrate and the tube wall are decoupled. In addition, we focus on the stick-slip motion of the contact line on the substrate. The energy dissipation function can be expressed as [40]

$$
\Phi=\frac{1}{2} \int_{R}^{R_{0}} 2 \pi r \frac{3 \eta}{h} v(r, t)^{2} d r+\frac{1}{2} 2 \pi R \xi_{c l} \dot{R}^{2}
$$


the first term denotes viscous dissipation in the lubrication approximation, and the second term denotes the extra dissipation of contact line movement on the substrate. Here, $\eta$ is the viscosity of the aqueous suspension, $\varepsilon$ is the molecular cut-off length of the order of $1 \mathrm{~nm}$, which is introduced to remove the divergence in the energy dissipation at the contact line [40]. And the velocity $v(r, t)$ in Eq. (5) is the height-average fluid velocity, calculated by the mass conservation equation of the solvent

$$
\frac{d}{d t} \int_{R}^{r} 2 \pi r^{\prime} h\left(r^{\prime}, t\right) d r^{\prime}=-2 \pi r v(r, t) h(r, t)-\pi\left(r^{2}-R^{2}\right) J(t) .
$$

Here, since the convection velocity is much faster than the diffusion velocity for macroscopic droplet, the diffusion of the solute in radical direction is ignored [41]. The solute transports along with the fluid, and then deposits on the substrate when it encounters the contact line.

By minimizing the Rayleighian $\mathfrak{R}$ with respect to $\dot{R}$, the evolution equation of $R$ can be obtained

$$
\left(1+k_{c l}\right) \dot{R}=-\frac{\left(R_{0}^{2}-R^{2}\right) \dot{V}}{4 R V}-\frac{\gamma_{L V} \theta\left[\left(\theta^{2}-\theta_{e}^{2}\right)+\frac{2 R_{0}^{2}}{R^{2}} \theta^{2}+\frac{R_{0}}{R} \theta\left(\theta_{e}^{2}-2\right)\right]}{6 \eta C},
$$

Where $C=R^{2} /\left(R_{0}^{2}-R^{2}\right)+\ln \left[\left(R_{0}^{2}-R^{2}\right) / 2 \varepsilon R\right]-2 R_{0}^{4} \ln \left(R_{0} / R\right) /\left(R^{2}-R_{0}^{2}\right)^{2}$. And $k_{c l}=\frac{\xi_{c l} \theta}{3 \eta C}$, which is related to the substrate wetting properties, substrate defects, and surface-active solutes $[42,43]$, denotes the importance of the extra friction constant $\xi_{c l}$ of the contact line relative to the normal hydrodynamic friction [44]. Hence, we assume that $k_{c l}$ changes with the contact angle [45] to mimic the stick-slip motion of the contact line: 


$$
k_{c l}= \begin{cases}0 & \text { for } \theta \leq \theta_{R} \text { or } \dot{\theta}>0, \\ 100 & \text { for } \theta>\theta_{R} \text { and } \dot{\theta} \leq 0 .\end{cases}
$$

To simplify the equation, the following three quantities are defined,

$$
\tau_{e v}=-\frac{V_{0}}{\dot{V}_{0}}, \tau_{r e}=\frac{\eta V_{0}^{\frac{1}{3}}}{\gamma_{L V} \theta_{e}^{3}}, \quad k_{e v}=\frac{\tau_{r e}}{\tau_{e v}}
$$

where $\tau_{e v}$ and $\tau_{r e}$ are respectively the characteristic time of evaporation and relaxation. $k_{e v}$ is the ratio of these two characteristic time. Therefore, Eq. (7) becomes

$$
\left(1+k_{c l}\right) \tau_{\mathrm{e} v} \dot{R}=\frac{\left(R_{0}^{2}-R^{2}\right)^{\frac{3}{2}} V_{0}}{4 R R_{0} V}-\frac{\theta V_{0}^{\frac{1}{3}}\left[\left(\theta^{2}-\theta_{e}^{2}\right)+\frac{2 R_{0}^{2}}{R^{2}} \theta^{2}+\frac{R_{0}}{R} \theta\left(\theta_{e}^{2}-2\right)\right]}{6 C k_{e v} \theta_{e}^{3}},
$$

Eqs. (1), (2), (8) and (10) determine the shape of restricted liquid during the entire event. The evolution equations of the system can be solved for given values of $k_{e v}, \theta_{r}$ and $\theta_{e}$. The equilibrium contact angle $\theta_{e}=0.58$ and the receding contact angle $\theta_{r}=0.35$ are used according to our experiments. We adopt $k_{e v}$ to be the usual value of 0.01 , which is proved in the previous work $[39,46]$. The restricted liquid shape evolution calculated from the theoretical model is shown in Figure 7, revealing the mechanisms of the formation of multi-ring pattern.

Figure 7 a shows that the motion of the contact line is similar to a staircase pattern, i.e. the typical stick-slip motion and the contact angle $\theta$ oscillates between the receding contact angle $\theta_{r}$ and another large one. The horizontal part of the staircase pattern means that the contact line is pinned, i.e. the stick state and the inclined part of the staircase pattern means that the contact line is depinned, i.e. the slip state. At the beginning, the contact line recedes and is pinned until $\theta$ 
increases to greater than $\theta_{r}$. Then, when $\theta$ decreases to $\theta_{r}$, the contact line begins to recede again until the next pinned state begins. After periodic stick-slip motion of contact line, multi-ring pattern forms. It's worth noting that the oscillation frequency of the contact angle increases and the pinning and depinning time of the contact line decrease with the evaporation proceeds. It shows that the evaporating rate increases with the reduction of liquid volume which is consistent with Eq. (3) indicating. Another thing to note that the contact angle when the contact line is pinned is not a constant, increasing with the evaporation proceeds. This change of contact angle is consistent with what is observed in the experiment, which is caused by the existence of the geometric container.

The position and spacing of rings are also analyzed. The theoretical prediction of ring positions agrees with the experimental results, as shown in Figure 7a. And Figure 7b shows that the ring spacing decreases exponentially with the ring radius from center to edge, which is quite consistent with the experimental results.

\section{CONCLUSION}

In this paper, the temperature effect of the substrate and the dimension effect of the geometric container on the formation of deposition patterns have been reported. We find that deposition patterns transition from honeycomb to multi-ring to island with $T$ increases and the dimension of the geometric container has an effect on the characteristics of patterns, that is, $D / L$ has an optimal range to form stable pattern and the ring-like pattern becomes more ordered and smoother as $L$ decreases. The mechanism controlling the formation of deposition patterns is the competition 
between $U_{P}$ and $U_{C L}$, and it is found that the competition between the two quantities is not monotonous as $T$ increases. The formation of the multi-ring pattern, which is mainly discussed in this paper, is attributed to the periodic stick-slip motion of the contact line and the particle sedimentation. And the spacing between neighboring rings decreases exponentially from center to edge. Moreover, considering the effects of internal flow field, the motion of contact line, and the solute transport, we develop a theoretical model based on the Onsager principle to reveal the physical mechanisms behind the multi-ring pattern phenomena. The theoretical predictions are in good agreement with the experiments. This may not only help to understand the formation of deposition patterns, but also assist future design and control in practical applications.

\author{
ASSOCIATED CONTENT \\ Supporting Information \\ Derivation of equations for the evolution of the restricted liquid during evaporation. \\ Videos of the stick-slip motion of the contact lines.
}

\title{
ACKNOWLEDGEMENT
}

This work was jointly supported by the National Natural Science Foundation of China (NSFC, Grant Nos. 11722223, 11672300 and 21822302), the Chinese Academy of Sciences Key Research Program of Frontier Sciences (Grant No. QYZDJ-SSW-JSC019), the Strategic Priority Research Program of the Chinese Academy of Sciences (Grant No. XDB22040401). 


\section{REFERENCES}

[1] Marín, Á. G.; Gelderblom, H.; Lohse, D.; Snoeijer, J. H. Order-to-disorder transition in ringshaped colloidal stains. Phys. Rev. Lett. 2011, 107, 085502.

[2] Yunker, P. J.; Still, T.; Lohr, M. A.; Yodh, A. G. Suppression of the coffee-ring effect by shape-dependent capillary interactions. Nature 2011, 476, 308-311.

[3] Alonso-Redondo, E.; Schmitt, M.; Urbach, Z.; Hui, C. M.; Sainidou, R.; Rembert, P.; Rembert, P.; Matyjaszewski, K.; Bockstaller, M. R.; Fytas, G. A new class of tunable hypersonic phononic crystals based on polymer-tethered colloids. Nat. Commun. 2015, 6, 8309.

[4] Derby, B. Inkjet printing of functional and structural materials: fluid property requirements, feature stability, and resolution. Annu. Rev. Mater. Res. 2010, 40, 395-414.

[5] Li, Y.; Lv, P.; Diddens, C.; Tan, H.; Wijshoff, H.; Versluis, M.; Lohse, D. Evaporationtriggered segregation of sessile binary droplets. Phys. Rev. Lett. 2018, 120, 224501.

[6] Alberts, E.; Warner, C.; Barnes, E.; Pilkiewicz, K.; Perkins, E.; Poda, A. Genetically tunable M13 phage films utilizing evaporating droplets. Colloid. Surface. B 2018, 161, 210-218.

[7] Lockett, M. R.; Smith, L. M. Fabrication and characterization of DNA arrays prepared on carbon-on-metal substrates. Anal. Chem. 2009, 81, 6429-6437.

[8] Ahmed, H.; Lee, L.; Darmanin, C.; Yeo, L. Y. A novel acoustomicrofluidic nebulization technique yielding new crystallization morphologies. Adv. Mater. 2018, 30, 1602040. 
[9] Walheim, S.; Bo, M.; Mlynek, J.; Krausch, G.; Steiner, U. Structure formation via polymer demixing in spin-cast films. Macromolecules 1997, 30, 4995-5003.

[10] Wang, J. W.; Li, B.; Cao, Y. P.; Feng, X. Q. Surface wrinkling patterns of film-substrate systems with a structured interface. J. Appl. Mech. 2015, 82, 051009.

[11] Wong, S.; Deubel, M.; Pérez-Willard, F.; John, S.; Ozin, G. A.; Wegener, M.; von Freymann, G. Direct laser writing of three-dimensional photonic crystals with a complete photonic bandgap in chalcogenide glasses. Adv. Mat. 2006, 18, 265-269.

[12] Cheng, W. L.; Zhang, W. W.; Chen, H.; Hu, L. Spray cooling and flash evaporation cooling: The current development and application. Renew. Sust. Energy Rev. 2016, 55, 614-628.

[13] Abbel, R.; Teunissen, P.; Michels, J.; Groen, W. A. Narrow conductive structures with high aspect ratios through single-pass inkjet printing and evaporation-induced dewetting. Adv. Eng. Mater. 2015, 17, 615-619.

[14] Talbot, E. L.; Yang, L.; Berson, A.; Bain, C. D. Control of the particle distribution in inkjet printing through an evaporation-driven sol-gel transition. ACS Appl. Mater. interfaces 2014, $6,9572-9583$.

[15] Deegan, R. D.; Bakajin, O.; Dupont, T. F.; Huber, G.; Nagel, S. R.; Witten, T. A. Contact line deposits in an evaporating drop. Phys.Rev. E 2000, 62, 756-765. 
[16] Deegan, R. D.; Bakajin, O.; Dupont, T. F.; Huber, G.; Witten, T. A. Capillary flow as the cause of ring stains from dried liquid drops. Nature 1997, 389, 827-829.

[17] Hu, H.; Larson, R. G. Marangoni effect reverses coffee-ring depositions. J. Phys. Chem. B 2006, 110, 7090-7094.

[18] Zhang, W.; Yu, T.; Liao, L.; Cao, Z. Ring formation from a drying sessile colloidal droplet. AIP Adv., 2013, 3, 102109.

[19] Chon, C. H.; Paik, S.; Tipton, J. B.; Kihm, K. D. Effect of nanoparticle sizes and number densities on the evaporation and dryout characteristics for strongly pinned nanofluid droplets. Langmuir 2007, 23, 2953-2960.

[20] Li, Y. F.; Sheng, Y. J.; Tsao, H. K. Solute concentration-dependent contact angle hysteresis and evaporation stains. Langmuir 2014, 30, 7716-7723.

[21] Chen, X.; Ma, R.; Li, J.; Hao, C.; Guo, W.; Luk, B. L.; Li, S. C.; Yao, S.; Wang, Z. Evaporation of droplets on superhydrophobic surfaces: surface roughness and small droplet size effects. Phys. Rev. Lett. 2012, 109, 116101.

[22] Zigelman, A.; Manor, O. The deposition of colloidal particles from a sessile drop of a volatile suspension subject to particle adsorption and coagulation. J. Colloid Interface Sci. 2017, 509, $195-208$

[23] Weon, B. M.; Je, J. H. Capillary force repels coffee-ring effect. Phys. Rev. E 2010, 82, 015305. 
[24] Gorr, H. M.; Zueger, J. M.; McAdams, D. R.; Barnard, J. A. Salt-induced pattern formation in evaporating droplets of lysozyme solutions. Colloids Surf., B 2013, 103, 59-66.

[25] Maheshwari, S.; Zhang, L.; Zhu, Y.; Chang, H. C. Coupling between precipitation and contact-line dynamics: Multiring stains and stick-slip motion. Phys. Rev. Lett. 2008, 100, 044503.

[26] Yabu, H.; Shimomura, M. Preparation of self-organized mesoscale polymer patterns on a solid substrate: Continuous pattern formation from a receding meniscus. Adv. Funct. Mater. 2005, $15,575-581$

[27] Xu, J.; Xia, J.; Hong, S. W.; Lin, Z.; Qiu, F.; Yang. Y. Self-assembly of gradient concentric rings via solvent evaporation from a capillary bridge. Phys. Rev. Lett. 2006, 96, 066104.

[28] Sun, W.; Yang, F. Q. Evaporation-assisted formation of deposition patterns from polymer solutions via copper tubes. eXPRESS Polym. Lett., 2018, 12, 699-712.

[29] Conway, J.; Korns, H.; Fisch, M. R. Evaporation kinematics of polystyrene bead suspensions. Langmuir 1997, 13, 426-431.

[30] Bormashenko, E.; Pogreb, R.; Stanevsky, O.; Bormashenko, Y.; Gendelman, O. Formation of honeycomb patterns in evaporated polymer solutions: Influence of the molecular weight. Mater. Lett. 2005, 59, 3553-3557. 
[31] Cui, L.; Han, Y. Honeycomb pattern formation via polystyrene/poly (2-vinylpyridine) phase separation. Langmuir 2005, 21, 11085-11091.

[32] Yang, X.; Li, C. Y.; Sun, Y. From multi-ring to spider web and radial spoke: competition between the receding contact line and particle deposition in a drying colloidal drop. Soft Matt. 2014, 10, 4458-4463.

[33] Maheshwari, S.; Zhang, L.; Zhu, Y.; Chang, H. C. Coupling between precipitation and contact-line dynamics: Multiring stains and stick-slip motion. Phys. Rev. Lett. 2008, 100, 044503.

[34] Zhang, S. F.; Luan, W. L.; Zhong, Q. X.; Yin, S. F.; Yang, F. Q. Evaporation-induced selfassembly of quantum dots-based concentric rings on polymer-based nanocomposite films. Soft Matt. 2016, 12, 8285-8296.

[35] Kim, H.; Boulogne, F.; Um, E.; Jacobi, I.; Button, E.; Stone, H. A. Controlled uniform coating from the interplay of Marangoni flows and surface-adsorbed macromolecules. Phys. Rev. Lett. 2016, $116,124501$.

[36] De Gennes, P. G. Instabilities during the evaporation of a film: Non-glassy polymer plus volatile solvent. Eur. Phys. J. E 2001, 6, 421-424.

[37] Kobayashi, M.; Makino, M.; Okuzono, T.; Doi, M. Interference effects in the drying of polymer droplets on substrate. J. Phys. Soc. Jpn. 2010, 79, 044802. 
[38] Parisse, F.; Allain, C. Drying of colloidal suspension droplets: experimental study and profile renormalization. Langmuir 1997, 13, 3598-3602.

[39] Doi, M. Soft Matter Physics; Oxford University Press, 2013.

[40] Man, X. K.; Doi, M. Ring to mountain transition in deposition pattern of drying droplets. Phys. Rev. Lett. 2016, 116, 066101.

[41] Anderson, D. M.; Davis, S. H. The Spreading of volatile liquid droplets on heated surfaces. Phys. Fluids 1995, 7, 248-265.

[42] Kajiya, T.; Monteux, C.; Narita, T.; Lequeux, F.; Doi, M. Contact-line recession leaving a macroscopic polymer film in the drying droplets of water-poly(N,N-dimethylacrylamide) (PDMA) solution. Langmuir 2009, 25, 6934-6939.

[43] Li, Y. F.; Sheng, Y. J.; Tsao, H. K. Evaporation stains: suppressing the coffee-ring effect by contact angle hysteresis. Langmuir 2013, 29, 7802-7811.

[44] Bonn, D.; Eggers, J.; Indekeu, J.; Meunier, J.; Rolley, E. Wetting and spreading. Rev. Mod. Phys. 2009, 81, 739-805.

[45] Wu, M. M.; Man, X. K.; Doi, M. Multi-ring deposition pattern of drying droplets. Langmuir 2018, 34, 9572-9578. 
[46] Kajiya, T.; Kobayashi, W.; Okuzono, T.; Doi, M. Controlling the drying and film formation processes of polymer solution droplets with addition of small amount of surfactants. $J$. Phys.

Chem. B 2009, 113, 15460-15466. 
(a)

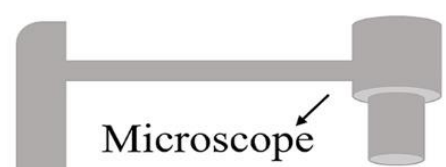

(b)

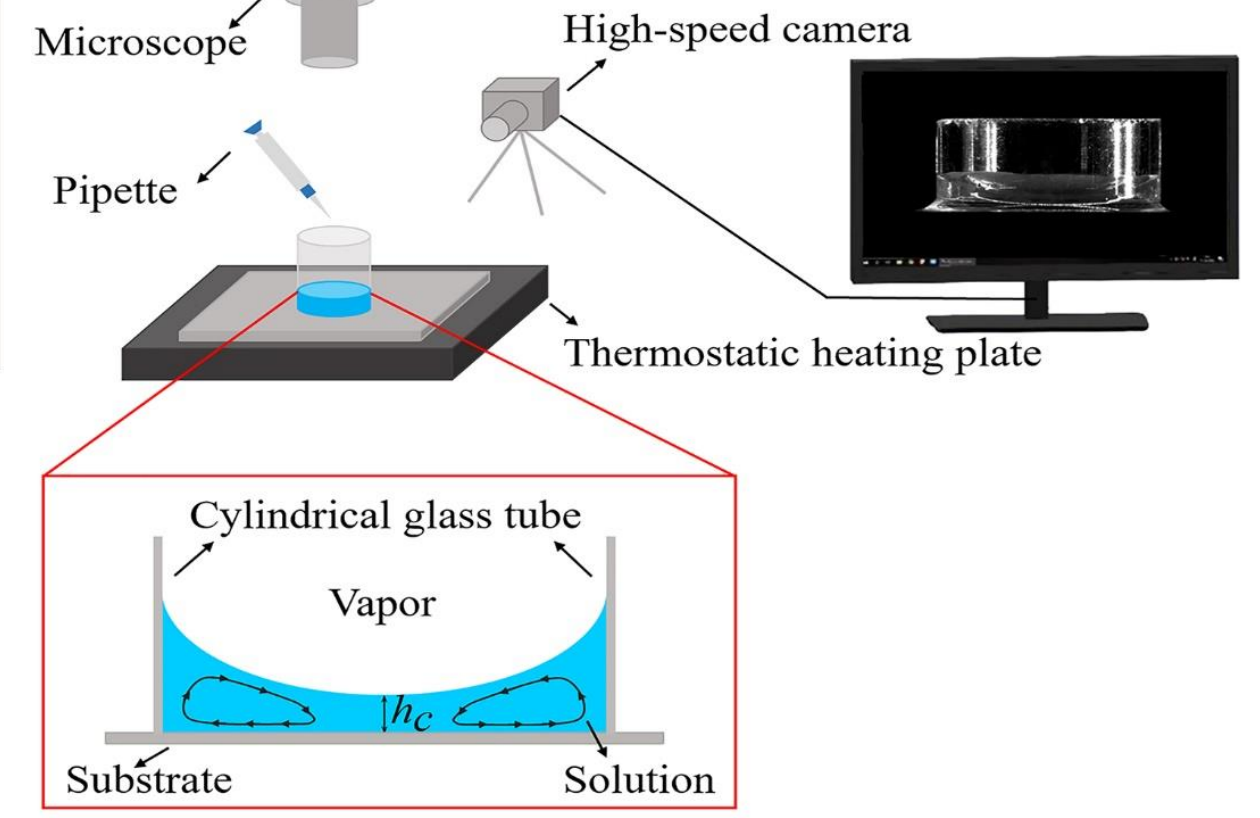

Figure 1. (a) Schematic of the experimental setup, (b) side view of the restricted liquid. 


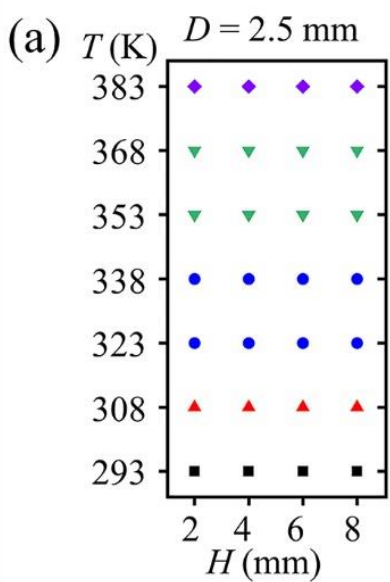

(b)

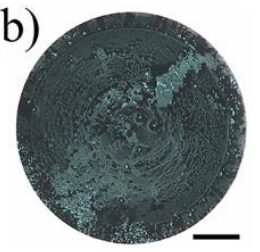

(c)

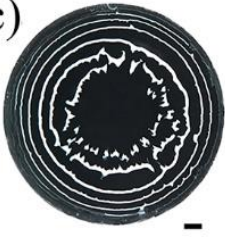

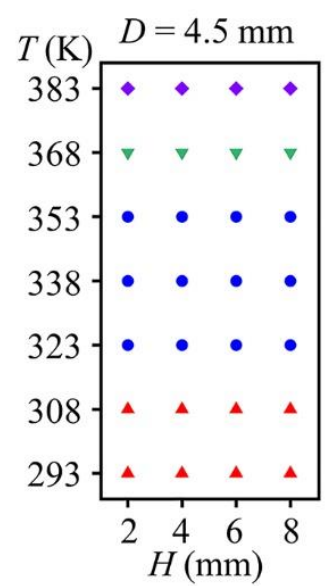

(d)

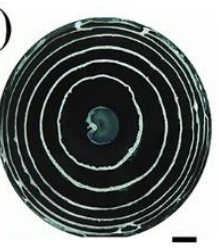

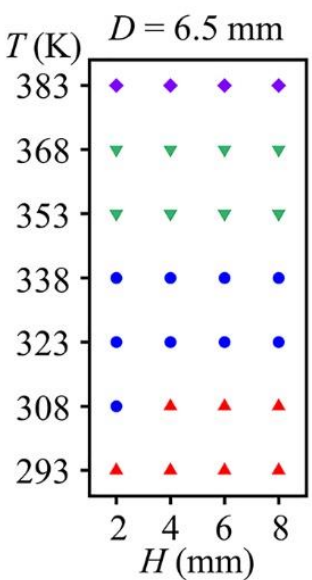

(e)

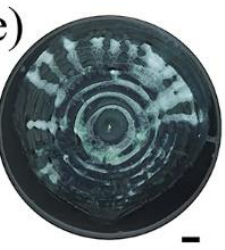

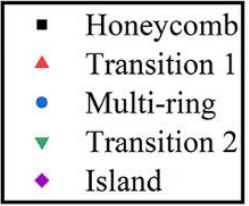

(f)

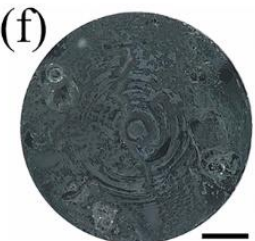

Figure 2. (a) Summary of the types of the deposition patterns formed with various geometric container dimensions, $D=2.5,4.5,6.5 \mathrm{~mm}$ and $L=2,4,6,8 \mathrm{~mm}$, and substrate temperatures, $T=$ 293 - 383 K. Optical imagines of deposition patterns (b) - (f) corresponds to honeycomb, transition 1, multi-ring, transition 2 and island. The scale bars are $0.5 \mathrm{~mm}$ in (b) - (f). 


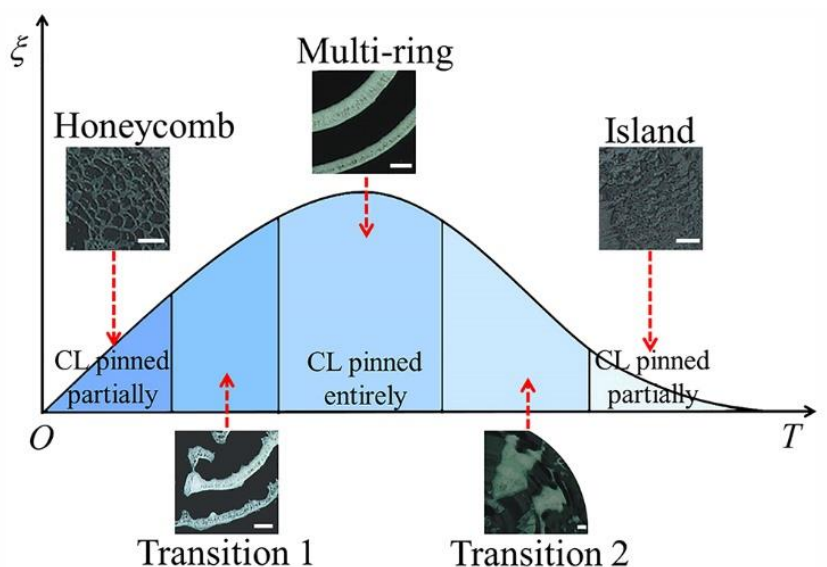

Figure 3. Deposition patterns transition in the case of $D=2.5 \mathrm{~mm}$ as a function of $T$ and a dimensionless parameter, $\xi=U_{P} / U_{C L}$, where $U_{P}$ and $U_{C L}$ are the deposition rate of particles and the contact line velocity, respectively. Note that Figure 3 is a schematic diagram of the transition of deposition patterns based on the experimental analysis. The scale bars are $0.1 \mathrm{~mm}$. 
(a)

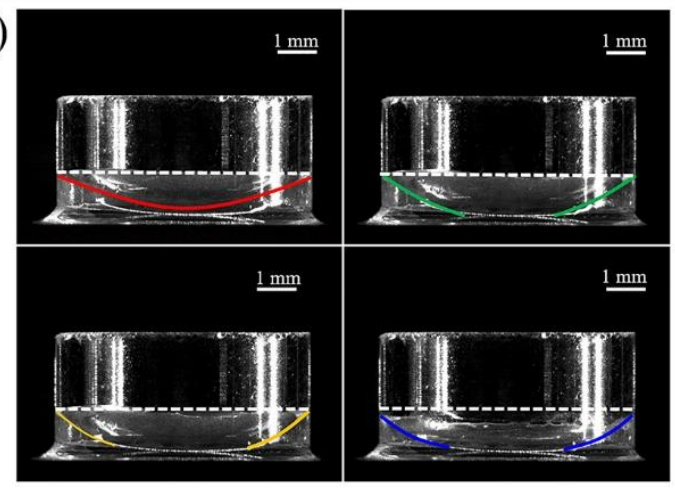

(b)

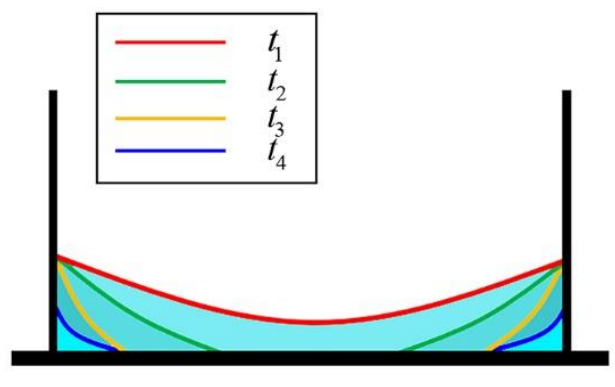

Figure 4. The evaporation process of restricted droplet. (a) The position of liquid-vapor interface at four transient time, $t_{1}<t_{2}<t_{3}<t_{4}$. Color solid lines and white dotted line represent liquid-vapor interface at different time and the highest position of liquid-vapor interface at the initial moment, respectively. (b) Schematic of the stick-slip motion on the substrate and tube wall based on (a). 
(a)

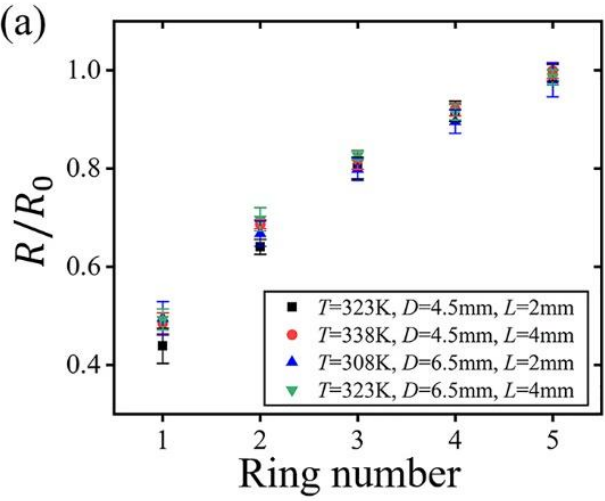

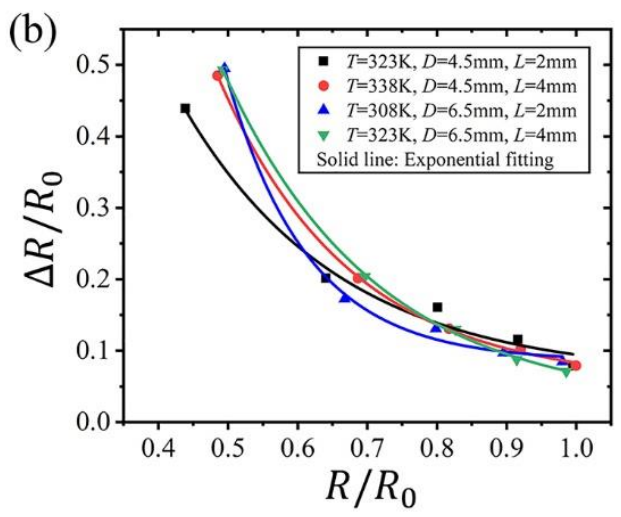

Figure 5. The experimental results for the typical multi-ring patterns at four different conditions. The variation of position (a) and spacing of rings (b) from center to edge. The data are fitted by an exponential function, $y \sim e^{-x}, \mathrm{x}$ and $\mathrm{y}$ represent dimensionless position and spacing of rings, respectively, showing that ring spacing decreases exponentially from center to edge. The innermost (whether solid or hollow) and outmost deposition are not included. 


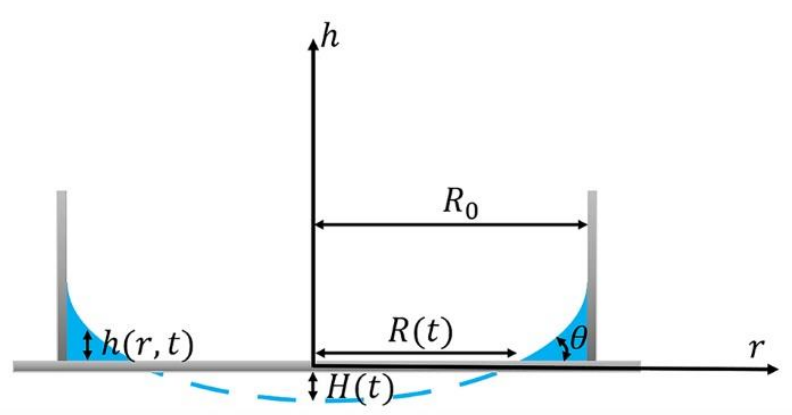

Figure 6. Schematic of the theoretical model. $R(t), h(r, t), \theta, R_{0}$ and $H(t)$ are the radius of the contact line, the profile of liquid-vapor interface, the contact angle, the radius of the tube and the height of the extension of the parabola at the center of the substrate, respectively. 
(a)

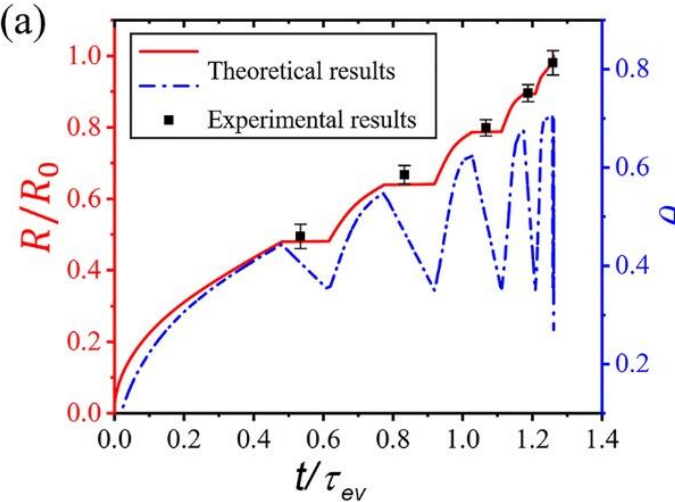

(b)

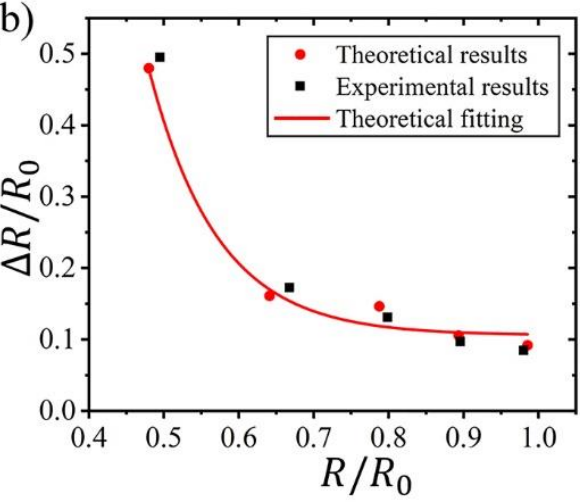

Figure 7. (a) The theoretical results for the evolution of the contact line and the contact angle during the evaporation process. The red solid and blue dash-dot lines represent the change of the contact line $R(t) / R_{0}$ and the contact angle $\theta$ with time $t / \tau_{e v}$, respectively. (b) The variation of ring spacing from center to edge and the red solid line is an exponential fitting curve of the theoretical results. The parameters are $k_{e v}=0.01, \theta_{r}=0.35, \theta_{e}=0.58$ and $\theta_{0}=0.02$. The black square is the experimental results based on the condition of $T=308 \mathrm{~K}, D=6.5 \mathrm{~mm}, L=2$ $\mathrm{mm}$, indicating the (a) position and (b) spacing of rings. 
TOC Graphic
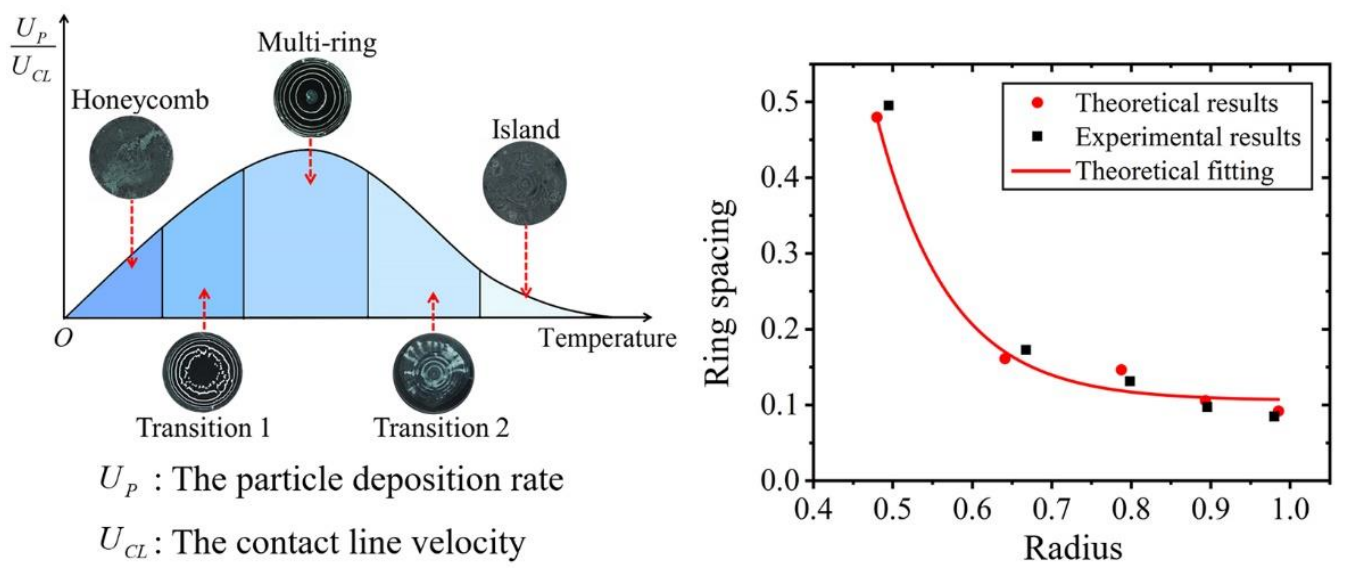

$U_{P}$ : The particle deposition rate

$U_{C L}$ : The contact line velocity 